\title{
BST2 Positive
}

National Cancer Institute

\section{Source}

National Cancer Institute. BST2 Positive. NCI Thesaurus. Code C154137.

An indication that BST 2 expression has been detected in a sample. 